\title{
Plants as a factory to produce molecules
}

\author{
Georges Freyssinet ${ }^{a}$ and Terry Thomas ${ }^{b}$ \\ ${ }^{2}$ Rhône-Poulenc, Scientific Direction, 25 Quai Paul Doumer, 92408 COURBEVOIE \\ Cédex, France. \\ ${ }^{\mathfrak{b}}$ Texas A \& M University, Department of Biology, College Station, Texas, U.S.A.
}

\begin{abstract}
Plants have been for many years the source of molecules of interest for Industry. One can cite, pharmaceutical molecules such as taxol or artemesin; starches and fatty acids for the Food and Chemical industries. The development of genetic engineering in plants offers new possibilities for improving the production of plant-made compounds or for the synthesis of new products in plants. In fact several applications have already been done, and the first crops are either in the market or close to the market. We will describe below several applications. In this direction, we have worked on the production of $\gamma$-linolenic acid (GLA), a fatty acid critical for human and animal health. GLA is currently produced from borage, evening primrose or black-currant. The production per hectare is low. If the cost of GLA production is reduced then new markets will be accessible. To reach this goal we have isolated a borage cDNA coding for a $\Delta 6-$ desaturase which transforms linoleic acid into GLA. This cDNA has been put under the control of various promoters and transferred into plants such as tobacco or Arabidopsis. GLA production up to $30 \%$ of the C-18 fatty acids has been obtained in leaves, and up to $10 \%$ in seeds.
\end{abstract}

\section{INTRODUCTION}

Plants have always been a source of molecules. In addition to being used for human and animal nutrition they have always brought other important and valuable compounds. Such compounds are used in various domains and one can cite for instance the production of pharmaceuticals, old ones such as morphine or more recent ones such as taxol. They have been used to produce polymers such as cellulose which is a versatile compound leading to many application, clothes, paper, linoleum.... In the food and industrial area several gums are extracted from plants: guar, carob. Plants are also a source of additives such as fatty acids used in soaps or formulation of active ingredients.

One of the main limitations for further use of molecules from plants has been the productivity due either to the fact that the plant has a low yield, a low content of the molecule or is grown in remote countries. With the development of new technologies such as genetic engineering, there has been increased interest in the use of plants as a source of novel molecules. In this short review, we will present the various approaches and some of the main achievements including the work done by our teams on the production of $\gamma$-linolenic acid (GLA).

\section{TECHNOLOGIES USED}

Source of new molecules: The main source of new molecules is still a direct search of new plants having useful properties. This is generally done by collecting plants in various environments, preparing extracts and evaluating the activity of these extracts in various tests. Such an approach is mainly used by the pharmaceutical industry. Native plants thus identified are either grown directly for production, or the active molecule is made through chemical synthesis. This has sometimes been a limiting step, and researchers have always tried to improve the production of the native plants (1). 
Improved productivity: In the past this has been mainly achieved by conventional plant breeding to improve the genetic background of the plant, its resistance to diseases and its ability to develop. Improved agronomic practices are also used for best productivity. When necessary, mutants have been produced to increase the genetic diversity and the production of the active molecules. However, the gain obtained is often limited mainly when one deals with complex secondary metabolites (2).

Genetic engineering: Since the development of genetic engineering in plants new perspectives appear. They consist either in the use of the technology to increase the endogenous production or to transfer the production of a given molecule into a crop giving higher yield. Genetic engineering allows the possibility to transfer new gene(s) in a plant cell and thus the insertion of a complete new pathway (3).

The approaches: Two approaches are used in the case of production of active molecules in plants. The gene transfer is either transient or stable. When it is transient the gene has to be injected in the plant at each generation, when it is stable the gene is inserted once and then remains in the plant throughout the generations. Both approaches have advantages and disadvantages.

The requirements: For genetic engineering three elements are critical, the gene(s), a system to transfer it into a plant cell and, in the case of stable insertion, a system to produce a fertile plant from the plant cell.

A gene: This is the crucial element, it brings the region coding for the protein of interest and the main sequences to control its expression. Figure 1 presents the various components of a gene. This gene is then inserted in a vector, alone or with other genes.

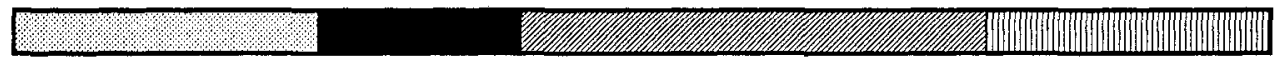

Promoter Coding Region Terminator

Targeting sequence Mature protein

Fig. 1: Structure of a transgene

The promoter controls the expression, it gives strength of expression and specificity (ubiquitous, inducible, tissue specific)

The coding region can be constituted of two parts:

- a targeting sequence which directs the protein to a cell compartment, e.g., chloroplast, mitochondria, vacuole;

- the region coding for the mature, active, protein.

The terminator controls expression and mainly its strength.

A transfer system: Depending on the approach, transient or stable, different transfer systems are available.

In the case of transient transfer, a viral vector is used. Most experiences have been gained with the tobacco mosaic vector (4). The gene of interest is inserted in a non-essential region of the virus genome. Then the virus is inoculated in individual host plants already growing. The plants are harvested after their growth, and the viral particles extracted. If the protein of interest is in the viral particles then they can be used directly. This approach is developed for instance to produce vaccines, the antigen is integrated in the viral coat protein to offer a good presentation of the epitopes (5). For protein production, the protein is not integrated in the viral particle, it is soluble in the cytoplasm of the plant cells, and it is isolated from the sap after removal of the viral particles. This approach gives generally high yield of the protein. It can be developed quickly since the only element needed is the gene for the product. The limiting factors are the fact that the inoculation has to be done each time and that we are dealing with crude extracts containing huge amounts of viral particles. 
In the case of stable transfer, the two techniques generally used are either the Biolistic ${ }^{\circledR}$ system or Agrobacterium. In the first case, naked DNA is embedded around small gold, tungsten or platinum particles which are shot inside the plant tissue using either powder or gas discharged systems. In the second case, the property of Agrobacterium to inject DNA into a plant cell is used. The gene(s) are placed between the natural borders of the transfer DNA present in the original plasmid of the bacteria. The plasmid obtained is transferred into the Agrobacterium. This Agrobacterium is cocultivated with the plant tissue. This natural vector has demonstrated its power, and several crop species, including monocotyledons, can now be transformed using these bacteria. This system gives cleaner insertion into the plant genome compared to the naked DNA system.

A system to regenerate a fertile plant: After the gene has been transferred in the plant cell then one needs to regenerate a fertile plant. Several approaches are available and will depend on the plant species. It is critical to be able to select the transformed cells early from the non-transformed tissue. To do that several selection procedures have been established. When a fertile plant is obtained then its progeny can be used for further analysis and production. One of the main limiting steps is the time needed to produce a good genetically modified plant (GMP), time to insert the gene and to evaluate the plants in normal agronomic environment. For more details on transformation technologies see ref. 3 .

\section{MAIN ACHIEVEMENTS}

In 1983 the first plant using genetic engineering was obtained, ten years later the first GMP reached the market place. Fairly early GMPs have been proposed for the production of new active molecules. Success has been obtained in various domains, if most of them are still at the research phase some have entered or are close to market. Table 1 below summarises some of the achievements.

TABLE 1: Main Achievements of GMPs to Produce Industrial Molecules

\begin{tabular}{lll}
\hline DOMAINS & PRODUCTS & COMPANIES/LABORATORIES \\
\hline Pharmaceuticals & Gastric lipase for cystic fibrosis & $\begin{array}{l}\text { Limagrain / Jouveinal (6) } \\
\text { CIBA / Univ. Calgary (6) }\end{array}$ \\
& $\begin{array}{l}\text { Hirudin as anticoagulant } \\
\text { Human haemoglobin }\end{array}$ & $\begin{array}{l}\text { Limagrain, INSERM Paris (7) } \\
\text { Pasteur Institute (8) }\end{array}$ \\
& Human epidermal growth factor & Several companies involved (5,9,10) \\
& Antigens / Antibodies & Several companies involved (5,11,12) \\
\hline Vaccines & Edibles vaccines & Several companies involved (13) \\
& Fatty acids composition and content & Several companies involved (14) \\
\hline Feed components & Improved content in lysine and methionine & Gist-Brocades / Mogen = Plant'zymes (15) \\
\hline Enzymes & Phytase & Calgene (16) \\
\hline Additives & Lauric acid & Several companies involved (17) \\
& New starch composition & Monsanto / Several laboratories (18) \\
\hline Polymers & Polyhydroxybutyrate & Several companies involved (19) \\
& Cellulose or lignin & Several companies involved (20) \\
\hline
\end{tabular}




\section{PRODUCTION OF $\gamma$-LINOLENIC ACID (GLA)}

GLA is a fatty acid important in human nutrition and has a number of medical and industrial applications (21). GLA is currently produced from either borage, evening primrose or black-currant. The yield of those plants is low and thus it would be beneficial if seed oils from a high yielding oilseed crop could be engineered to produce GLA. GLA is obtained through the transformation, by a $\Delta 6-$ desaturase, of linoleic acid, a C18:2 present in large amount in most oilseed crops. A cDNA coding for a $\Delta 6$-desaturase was isolated from the ornamental plant Borago officinalis using expressed sequence tag analysis. This gene is more similar to the $\Delta 6$-desaturase gene isolated before from Synechocystis (22) than with other desaturases (23). This cDNA has been put under the control of various promoters, constitutive or seed specific. The chimaeric genes obtained were introduced into tobacco or Arabidopsis using Agrobacterium. Genetically modified plants were analysed for their C:18 composition by gas chromatography. A peak corresponding to the retention time of GLA was observed in extracts from genetically modified plants (23). Table 2 summarises the data obtained

TABLE 2 . Expression of the borage $\Delta-6$ desaturase in genetically modified plants

\begin{tabular}{lllrll}
\hline Promoter & Plant & \multicolumn{2}{c}{ Seed } & \multicolumn{2}{c}{ Leaf } \\
\cline { 3 - 6 } & & GLA & Range & GLA $^{*}$ & Range \\
\hline Constitutive, CaMV 35S & Tobacco & 1.3 & $0.7-3.1$ & 20 & $19-22$ \\
\hline Seed specific, Arabidospsis oleosin & Arabidopsis & 3.1 & $0-6.7$ & n.d. & \\
\hline Seed specific, Sunflower albumin & Arabidopsis & 4.4. & $3.1-10.2$ & n.d. & \\
\hline
\end{tabular}

* mean value expressed as the percent of the $\mathrm{C} 18$ fatty acids

n.d., not detected

These results clearly demonstrate that production of GLA in a new plant species is possible, the level achieved in seeds is similar to the GLA content of evening primrose oil. The transfer of the gene in various crops such as canola, flax, sunflower, soybean or maize can bring new sources of GLA which can be used in various applications such as human and animal food, pharmaceuticals, cosmetics and possibly as industrial feed stocks.

\section{LIMITATIONS AND FUTURE PROSPECTS}

We have shown above that improved production of a product already made in a plant or the production of an entirely new product can be achieved in plants using genetic engineering. A few examples are on the market, canola rich in lauric acid, several are close to market, high lysine and methionine seeds, high oleic canola or soybean. However, several limitations have still to be overcome:

\section{Limitations:}

Stability of the transgene: It has been shown that transgenes inserted in the nuclear plant genome are stable and transferred from one generation to the other with conventional Mendelian heritability. However, the expression of the transgene is sometimes unstable and co-suppression may occur (24). This happens generally during the first generation after transformation, and thus plants showing this phenomenon can be discarded. It may be a problem when homologous genes are inserted in the plant genome.

Extraction and purification: When the product has been made in the plant most of the time extraction and purification will be required. Depending on the molecule and its use these steps may be limiting. For molecules already extracted from plants such as starch, fatty acids, or secondary metabolites, techniques are already available. An increase in the content of the molecule should help in the purification process. For new molecules and mainly for proteins it may be difficult to obtain samples with $100 \%$ purity. For some applications impurities may not be a problem, for others, and 
mainly in the vaccine or pharmaceutical areas, this may pose difficulties. New technologies will have to be developed to avoid contamination by extraneous proteins, DNA, polysaccharides or lipids. It is noteworthy that for certain applications the use of the whole plants is envisaged, this is for instance the case for phytase where MOGEN intends to use canola seeds enriched in phytase to feed animals. This is also proposed for some applications in the vaccination area where, for instance, fruits containing the antigen can be used directly for vaccination. Several groups are developing plants producing antigens and are using the plant tissue, a banana or a tomato for example, as a vehicle for the vaccine. In these cases, no extraction or purification of the active compound is needed.

Conformity of the molecule: Several molecules, mainly proteins, undergo post-translational modification, glycosylation, phosphorylation. Such modifications may not be well reproduced in the plants. To overcome this defect, several groups are looking at ways to integrate in the plant systems which allow a correct maturation of protein, conforming to the original one. This may be critical for the production of pharmaceutical proteins in plants.

Environmental impact: The plant will be grown in the nature and the potential impact on the environment should be carefully evaluated. It is not obvious that such plants will have a better fit with the environment and thus may develop as weeds. The only concern may be the transfer of pollen to relatives. In this case one should evaluate the potential risk linked to the new trait introduced in the crop. If a potential risk is identified appropriate measures to isolate the crops, as is normally done by breeders and seed companies may be required.

\section{Future prospects:}

Despite these few limitations the use of crops as a factory to produce molecules will expand. Crops are already used for several products and thus expertise is available to solve most of the above limitations, e.g., extraction and purification, environmental impact. A canola rich in lauric acid has been in the market for two years, and its further development is expected. Results published in the literature indicate that others plants are ready for market and that the number will increase with time. The most obvious ones are plants which are already used for production of pharmaceuticals, alkaloids for instance, these are also the most difficult ones since one has to modify complete biochemical pathways. Success in the isolation of enzymes for such pathways indicates that improved plants will be possible. The first major development will be with system where one gene is sufficient for the production of the compound, this is the case for production of proteins, phytase, lipase, fatty acid biosynthesis when one gene changes the composition, e.g., lauric acid, GLA (see also 25). With the plants available we see only the top of the iceberg and as time and knowledge develop its size will undoubtedly increase.

\section{REFERENCES}

1. P. Houghton. Agro-Food-Industry Hi-Tech 6, 12-16 (1995).

2. D.D. Ellis, E.L. Zeldin, M. Brodhagen, W.A. Russin, and B.H. McCown. J. Nat. Prod. 59, 246250 (1996).

3. K.P. Pauls. Biotechnology Adavances 13, 673-693 (1995).

4. T.H. Turpen, S.J. Reinl, Y. Sharoenvit, S.L. Hoffmann, V. Fallarme and Grill L.K. Bio/Technology 13, 53-57 (1995).

5. H.S. Mason and C.J. Arntzen. TIBTECH 13, 388-392 (1995).

6. V. Gomord, J.P. Salier et L. Faye. Biofutur 154, 26-30 (1996).

7. W. Dieryck, J. Pagnier, C. Poyart, M.C. Marden, V. Gruber, P. Bournat, S. Baudino and B. Mérot. Nature 386, 29-30 (1997).

8. A.H. Salmanian, A. Gushchin, T. Medvedeva, M.R. Noori-Daloii and N. Domansky. Biotechnology Letters 18, 1095-1098 (1996).

9. J.K.C. Ma, A. Hiatt, M. Hein, N.D. Vine, F. Wang, P. Stabila, C. van Dolleweerd, K. Mostov and T. Lehner. Science 268, 716-719.

10. U. Fielder and U. Conrad. Bio/Technology 13, 1090-1093.

11. K. Dalsgaard, A. Uttenthal, T.D. Jones, F. Xu, A. Merryweather, W.D.O. Hamilton, J.P.M. Langeveld, R.S. Boshuizen, S. Kamstrup, G.P. Lomonossoff, C. Porta, C. Vela, J.I. Casal, R.H. Meloen and P.B. Rodgers. Nature Biotech. 15, 248-252 (1997). 
12. P.B. McGarvey, J. Hammond, M.M. Dienelt, D.C. Hooper, Z.F. Fu, B. Dietzschold, H. Koprowski and F.H. Michaels. Bio/Technology 13, 1484-1487 (1995).

13. R. Töpfer, N. Martini and J. Schell. Science 268, 681-686 (1995).

14. S.C. Falco, T. Guida, M. Locke, J. Mauvais, C. Sanders, R.T. Ward and P. Webber. Bio/Techn. 13, 577-582 (1995).

15. J. Pen. Agro-Food-Industry Hi-Tech. 8, 9-13, (1996).

16. T.A. Voelker, A.C. Worrell, L. Anderson, J. Bleibaum, C. Fan, D.J. Hawkins and S.E. Radke. Science 257, $72-74$ (1992)

17. C.K. Shewmaker, C.D. Boyer, D.P. Wiesenborn, D.B. Thompson, M.R. Boersig, J.V. Oakes and D.M. Stalker. Plant Physiol, 104, 1159-1166 (1992).

18. C. Nawrath, Y. Poirier and C. Somerville. Proc. Natl. Acad. Sci. USA. 91, 12760-12764 (1994).

19. U.V. Dwivedi, W.H. Campbell, J. Yu, R.S.S Datla, R.C. Bugos, V.L. Chaing and G.K. Podila. Plant Molec. Biol., 26, 61-71 (1994).

20. M.W. Lassner, C.K. Levering, H.M. Davies and D.S. Knutzon. Plant Physiol., 109, 1389-1394 (1995).

21. R.R. Brenner. Adv. Exp. Med. Biol. 83, 85-101 (1976).

22. A.S. Reddy and T.L. Thomas. Nature Biotechnology 14, 639-642 (1996).

23. A.N. Nunberg, P.D. Beremand and T.L. Thomas. In preparation.

24. M.A. Matzke and A.J.M. Matzke. Plant Physiol. 107, 679-685 (1995).

25. O.J.M. Goddijn and J. Pen. TIBTECH 13, 379-387 (1995). 